\title{
Cantor's intersection theorem for $K$-metric spaces with a solid cone and a contraction principle
}

\author{
Jacek Jachymski and Jakub Klima
}

\author{
With great respect and admiration for Professor Andrzej Granas
}

\begin{abstract}
We establish an extension of Cantor's intersection theorem for a $K$-metric space $(X, d)$, where $d$ is a generalized metric taking values in a solid cone $K$ in a Banach space $E$. This generalizes a recent result of Alnafei, Radenović and Shahzad (2011) obtained for a $K$-metric space over a solid strongly minihedral cone. Next we show that our Cantor's theorem yields a special case of a generalization of Banach's contraction principle given very recently by Cvetković and Rakočević (2014): we assume that a mapping $T$ satisfies the condition " $d(T x, T y) \preceq \Lambda(d(x, y))$ " for $x, y \in X$, where $\preceq$ is a partial order induced by $K$, and $\Lambda: E \rightarrow E$ is a linear positive operator with the spectral radius less than one. We also obtain new characterizations of convergence in the sense of Huang and Zhang in a $K$-metric space.
\end{abstract}

Mathematics Subject Classification. Primary 47H09, 47H10, 54H25; Secondary 46B40, 54E35.

Keywords. $K$-metric space, cone metric space, solid cone, Cantor's intersection theorem, fixed point, spectral radius, contraction principle.

\section{Introduction}

It is well known that the classical Banach fixed point principle can be derived from Cantor's intersection theorem. This observation is due to Boyd and Wong [3] and their proof can also be found in [10, p. 8] or [11, p. 2]. Actually, Cantor's theorem has a number of applications in fixed point theory; see, e.g., the papers of Dugundji [8] on positive definite functions, Goebel [9] on nonexpansive mappings, Kirk [22] on asymptotic contractions, Jachymski [16] on generalized contractions, or the monograph of Granas and Dugundji [12, pp. 17 and 27], where, in particular, an elegant proof of the Bishop-Phelps theorem is given. 
On the other hand, Granas and Horvath [13] (see also [12, pp. 3233]) established the so-called order-theoretic Cantor theorem, from which they derived a lot of results related to the Bishop-Phelps theorem. Recently, another extension of Cantor's theorem was given by Alnafei, Radenović and Shahzad [2], who used it to obtain a fixed point theorem for mappings on $K$-metric spaces. Recall that a $K$-metric space is a pair $(X, d)$, where $X$ is a nonempty set and $d$ is a vector-valued function from $X \times X$ to a closed cone $K$ in a Banach space $E$, satisfying three well-known axioms of a metric with respect to the following partial order in $E$ : for $a, b \in E$,

$$
a \preceq b \quad \text { if and only if } \quad b-a \in K \text {. }
$$

This notion was first introduced in 1934 by Kurepa [24], who used the term "pseudodistance" for $d$. Following Zabrelko we, however, use the term " $K$ metric" instead of "pseudodistance" since now the latter term has a different meaning. $K$-metric spaces were rediscovered in 2007 by Huang and Zhang [15] under the name "cone metric spaces." They also established an extension of Banach's contraction principle for mappings $T$ satisfying the following condition:

$$
d(T x, T y) \preceq \lambda d(x, y) \quad \text { for } x, y \in X,
$$

where $\lambda \in[0,1)$. Subsequently, their result was generalized by a number of authors; see, e.g., the survey paper [19] and 100 references therein.

Our purpose here is to establish an extension of Cantor's theorem for $K$-metric spaces. Our result is more general than that given in [2] since we omit the extra assumption used in [2] that a cone $K$ is strongly minihedral, i.e., every subset of $E$ which is bounded from above with respect to the partial order $\preceq$ defined by (1.1) has a supremum; cf., e.g., [5, p. 219]. Moreover, our proof is completely different: instead of adapting an argument from the classical proof as done in [2], we use a remetrization technique. This is preceded by results on a characterization of some types of convergence in a Banach space $E$, induced by a solid cone $K$, i.e., the cone with a nonempty interior. Hence, as an immediate consequence, we also get a characterization of convergence in the sense of Huang and Zhang [15] in a $K$-metric space. At last, with the help of our Cantor's theorem, we prove an extension of Banach's contraction principle for mappings $T$ satisfying the condition

$$
d(T x, T y) \preceq \Lambda(d(x, y)) \quad \text { for } x, y \in X,
$$

where $\Lambda: E \rightarrow E$ is a linear bounded operator, which is positive, i.e., $\Lambda(K) \subseteq$ $K$, and $r(\Lambda)$, the spectral radius of $\Lambda$, is less than one. Clearly, (1.2) is a particular case of (1.3) with $\Lambda:=\lambda I$, where $I$ is the identity mapping on $E$. Contractive conditions of type (1.3) were studied by many mathematicians, mainly from the former Soviet Union, starting from the paper of Perov [25], in which the case when $E=\mathbb{R}^{m}$ was considered. A more general fixed point theorem for such mappings may be found in the monograph of Krasnosel'skiu et al. [23, pp. 91-92]. Here a cone $K$ is assumed to be normal, i.e.,

$$
\inf \{\|x+y\|: x, y \in K,\|x\|=\|y\|=1\}>0 .
$$


In our theorem we allow $K$ to be nonnormal, however, $K$ is assumed to be solid. In fact, as pointed out to us by the referee, the theorem is a special case of the result obtained very recently by Cvetković and Rakočević [4], who considered a more general contractive condition than (1.3). However, our argument used in the proof is completely different from that in [4].

Extensions of Banach's contraction principle using conditions of type (1.3) have many interesting applications to functional or differential equations. In particular, in our opinion, the most elegant proof of the classical Picard-Lindelöf theorem is that one which uses Perov's fixed point theorem. The details of this proof can be found in our recent paper [18]; we were inspired by the paper [6]. In fact, the original proof is probably given in Perov's paper [25], however, it seems that this article is now unavailable.

\section{On $c$-uniform convergence in $E$ and the Huang-Zhang convergence in $X$}

Throughout this section we assume that $E$ is a Banach space and $K$ is a solid cone in $E$, i.e., int $K \neq \emptyset$. Then the ordered Banach space $E$ is called a Krein space (see, e.g, [1, Definition 2.62]).

Let $(X, d)$ be a $K$-metric space, i.e., $d: X \times X \rightarrow K$ satisfies the three axioms of a metric with respect to the partial order $\preceq$ defined by (1.1). For $a, b \in E, a \ll b$ stands for $b-a \in \operatorname{int} K$, and $a \prec b$ means that $a \preceq b$ and $a \neq b$. Huang and Zhang [15] introduced the following definition of convergence in $(X, d)$ : a sequence $\left(x_{n}\right)$ is $d$-convergent to $x \in X$, if for any $c \gg 0$, there is $k \in \mathbb{N}$ such that

$$
d\left(x_{n}, x\right) \ll c \quad \text { for } n \geq k \text {. }
$$

We denote it by

$$
x_{n} \stackrel{d}{\longrightarrow} x .
$$

In a similar way, as in a metric setting, they defined Cauchy's sequences and then the completeness of $(X, d)$. Recently, Du [7] and Kadelburg, Radenović and Rakočević [20] have proved (in different ways) that there exists a realvalued metric $\rho$ on $X$ such that for any sequence $\left(x_{n}\right),\left(x_{n}\right)$ is $d$-convergent if and only if it is $\rho$-convergent, and $\left(x_{n}\right)$ is a $d$-Cauchy sequence if and only if it is a $\rho$-Cauchy sequence. In particular, it means that the topology induced by $d$ on $X$ via the limit operator is metrizable.

In this section we propose yet another approach to the problem of metrizability of $(X, d)$. Namely, first we give a characterization of some type of convergence in a Banach space $E$. Then, as an immediate consequence, we get the above metrizability result as well as other equivalent conditions for the convergence in the sense of Huang and Zhang in $(X, d)$.

Given $a, b \in E$ with $a \preceq b$, we denote by $[a, b]$ the order interval, i.e.,

$$
[a, b]:=\{c \in E: a \preceq c \preceq b\} .
$$


For $c \in K$, the ideal generated by $c$ (see [1, Definition 2.53]) is the linear subspace

$$
E_{c}:=\bigcup_{n \in \mathbb{N}}[-n c, n c] .
$$

We can define the Minkowski functional on $E_{c}$ by setting

$$
\|a\|_{c}:=\inf \{\lambda>0: a \in[-\lambda c, \lambda c]\} \quad \text { for } a \in E_{c} .
$$

For the following result, see, e.g., [1, Theorem 2.55] and take into account that $E$ is Archimedean (see [1, Definition 1.10]).

Theorem 2.1. For any $c \in K$, we have the following.

(1) The Minkowski functional $\|\cdot\|_{c}$ is a monotone norm on $E_{c}$.

(2) The closed unit ball in $\left(E_{c},\|\cdot\|_{c}\right)$ coincides with the order interval $[-c, c]$.

(3) The cone $K \cap E_{c}$ is $\|\cdot\|_{c}$-closed.

Now if $c \in \operatorname{int} K$, then by [1, Lemma 2.5], $0 \in \operatorname{int}[-c, c]$, so $[-c, c]$ is absorbing, and hence $E_{c}=E$. Thus Theorem 2.1 and [27, Theorem 1.36] yield the following corollary.

Corollary 2.2. Let $K$ be a solid cone in a Banach space $(E,\|\cdot\|)$ and let $c \in \operatorname{int} K$. Then the Minkowski functional $\|\cdot\|_{c}$ is a monotone norm on $E$ and is continuous with respect to the norm $\|\cdot\|$. Moreover, $K$ is $\|\cdot\|_{c}$-closed and

$$
[-c, c]=\left\{a \in E:\|a\|_{c} \leq 1\right\} .
$$

Remark 2.3. Let us observe that the Minkowski functional $\|\cdot\|_{c}$ is a norm on $E$ only if $c \in \operatorname{int} K$. Indeed, if it is a norm on $E$, then $E_{c}=E$, so by [1, Lemma $2.54(\mathrm{~b})] c$ is an order unit of $E$ (cf. the definition in [1, p. 5]) and by [1, Theorem 2.8], $c$ is an interior point of $K$.

Now let $c \succ 0$ and $\left(a_{n}\right)$ a sequence in $E$. Following [1, Definition 2.56] we say that $\left(a_{n}\right)$ is c-uniformly convergent to $a \in E$ (in symbols, $a_{n} \stackrel{c}{\rightarrow} a$ ), if for any $\varepsilon>0$, there is $k \in \mathbb{N}$ such that

$$
-\varepsilon c \preceq a_{n}-a \preceq \varepsilon c \text { for } n \geq k .
$$

Lemma 2.4. $a_{n} \stackrel{c}{\rightarrow} a$ if and only if $a_{n}-a \in E_{c}$ for sufficiently large $n$ and $\left\|a_{n}-a\right\|_{c} \rightarrow 0$.

Proof. Observe that the condition $-\varepsilon c \preceq a_{n}-a \preceq \varepsilon c$ is equivalent to $(1 / \varepsilon)\left(a_{n}-a\right) \in[-c, c]$, which in turn means in view of Theorem 2.1(2) that

$$
\left\|\frac{1}{\varepsilon}\left(a_{n}-a\right)\right\|_{c} \leq 1,
$$

i.e., $\left\|a_{n}-a\right\|_{c} \leq \varepsilon$. This yields the result.

Inspired by the Huang-Zhang definition, we say that a sequence $\left(a_{n}\right)$ in $E$ with a solid cone $K$ is $K$-convergent to $a \in E$ (in symbols, $a_{n} \stackrel{K}{\longrightarrow} a$ ), if for any $c \gg 0$, there is $k \in \mathbb{N}$ such that

$$
-c \ll a_{n}-a \ll c \text { for } n \geq k \text {. }
$$


Below we give a list of equivalent conditions for this convergence. The last of them is related to the notion of a fundamental sequence in the Kantorovich sense (see [28, p. 830]).

Theorem 2.5. Let $K$ be a solid cone in a Banach space $E,\left(a_{n}\right)$ a sequence in $E$ and $a \in E$. The following statements are equivalent:

(i) $a_{n} \stackrel{K}{\longrightarrow} a$;

(ii) for any $c \gg 0$, there is $k \in \mathbb{N}$ such that

$$
-c \preceq a_{n}-a \preceq c \quad \text { for } n \geq k ;
$$

(iii) for any $c \gg 0$, there is $k \in \mathbb{N}$ such that

$$
\left\|a_{n}-a\right\|_{c} \leq 1 \quad \text { for } n \geq k
$$

(iv) for any $c \gg 0, a_{n} \stackrel{c}{\longrightarrow} a$;

(v) for any $c \gg 0,\left\|a_{n}-a\right\|_{c} \rightarrow 0$;

(vi) there exists $c \gg 0$ such that $a_{n} \stackrel{c}{\longrightarrow} a$;

(vii) there exists $c \gg 0$ such that $\left\|a_{n}-a\right\|_{c} \rightarrow 0$;

(viii) there exists a sequence $\left(c_{n}\right)$ in $K$ such that $\left\|c_{n}\right\| \rightarrow 0$ and

$$
-c_{n} \preceq a_{n}-a \preceq c_{n} \quad \text { for any } n \in \mathbb{N} \text {. }
$$

Proof. (i) $\Rightarrow$ (ii) is obvious since $a \ll b$ implies $a \preceq b$ for $a, b \in E$.

(ii) $\Leftrightarrow$ (iii) follows immediately from Corollary 2.2 , by which the condition " $-c \preceq a_{n}-a \preceq c$ " is equivalent to " $\left\|a_{n}-a\right\|_{c} \leq 1$."

(ii) $\Rightarrow$ (iv): Let $c \gg 0$ and $\varepsilon>0$. Then $\varepsilon c \gg 0$, so by (ii), there is $k \in \mathbb{N}$ such that $-\varepsilon c \preceq a_{n}-a \preceq \varepsilon c$ for $n \geq k$. That means (iv) holds.

(iv) $\Leftrightarrow(\mathrm{v})$ and (vi) $\Leftrightarrow$ (vii) follow from Lemma 2.4.

(iv) $\Rightarrow$ (vi) is obvious.

(vi) $\Rightarrow$ (viii): By (vi), there is $c \gg 0$ such that $a_{n} \stackrel{c}{\longrightarrow} a$. Hence there exists an increasing sequence $\left(k_{n}\right)$ of positive integers such that

$$
-\frac{1}{n} c \preceq a_{j}-a \preceq \frac{1}{n} c \quad \text { for } j>k_{n} .
$$

We have that for $j=1, \ldots, k_{1}, a_{j}-a \in E=\bigcup_{n \in \mathbb{N}}[-n c, n c]$, so there is $p \in \mathbb{N}$ such that

$$
-p c \preceq a_{j}-a \preceq p c \quad \text { for } j=1, \ldots, k_{1} \text {. }
$$

Set $c_{n}:=p c$ for $n=1, \ldots, k_{1}$, and $c_{n}:=(1 / m) c$ for $m \in \mathbb{N}$ and $n \in \mathbb{N}$ with $k_{m}<n \leq k_{m+1}$. It is easily seen that $\left(c_{n}\right)$ has the properties as in (viii).

(viii) $\Rightarrow\left(\right.$ i): Let $\left(c_{n}\right)$ be as in (viii). Fix $c \gg 0$. Then $(1 / 2) c \gg 0$, so $0 \in \operatorname{int}[(-1 / 2) c,(1 / 2) c]$. Hence, since $\left\|c_{n}\right\| \rightarrow 0$, there is $k \in \mathbb{N}$ such that 
$(-1 / 2) c \preceq c_{n} \preceq(1 / 2) c$ for $n \geq k$, so by (viii), we obtain that

$$
-c \ll-\frac{1}{2} c \preceq a_{n}-a \preceq \frac{1}{2} c \ll c \quad \text { for } n \geq k .
$$

Thus (i) holds.

Now let $(X, d)$ be a $K$-metric space with a solid cone $K$. For any $c \gg 0$, set

$$
\rho_{c}(x, y):=\|d(x, y)\|_{c} \quad \text { for } x, y \in X .
$$

It follows from Corollary 2.2 that $\rho_{c}$ is a (real-valued) metric on $X$. Since the Huang-Zhang convergence of a sequence $\left(x_{n}\right)$ to $x \in X$ means that

$$
d\left(x_{n}, x\right) \stackrel{K}{\longrightarrow} 0
$$

Theorem 2.5 yields the following corollary.

Corollary 2.6. Let $(X, d)$ be a $K$-metric space over a solid cone $K,\left(x_{n}\right)$ a sequence in $X$ and $x \in X$. The following statements are equivalent:

(i) $x_{n} \stackrel{d}{\longrightarrow} x$ (the Huang-Zhang convergence);

(ii) for any $c \gg 0$, there is $k \in \mathbb{N}$ such that

$$
d\left(x_{n}, x\right) \preceq c \quad \text { for } n \geq k ;
$$

(iii) for any $c \gg 0$, there is $k \in \mathbb{N}$ such that

$$
\rho_{c}\left(x_{n}, x\right) \leq 1 \quad \text { for } n \geq k ;
$$

(iv) for any $c \gg 0, d\left(x_{n}, x\right) \stackrel{c}{\rightarrow} 0$;

(v) for any $c \gg 0, \rho_{c}\left(x_{n}, x\right) \rightarrow 0$;

(vi) there exists $c \gg 0$ such that $d\left(x_{n}, x\right) \stackrel{c}{\rightarrow} 0$;

(vii) there exists $c \gg 0$ such that $\rho_{c}\left(x_{n}, x\right) \rightarrow 0$;

(viii) there exists a sequence $\left(c_{n}\right)$ in $K$ such that $\left\|c_{n}\right\| \rightarrow 0$ and

$$
d\left(x_{n}, x\right) \preceq c_{n} \quad \text { for any } n \in \mathbb{N} .
$$

Corollary 2.6 shows that a $K$-metric $d$ is equivalent to the metric $\rho_{c}$ for any $c \gg 0$. It turns out that $d$ and $\rho_{c}$ are also Cauchy equivalent (i.e., every $d$-Cauchy sequence is a $\rho_{c}$-Cauchy sequence and vice versa) as stated in the following corollary.

Corollary 2.7. Let $(X, d)$ be a $K$-metric space over a solid cone $K$ in $E$ and $\left(x_{n}\right)$ a sequence in $X$. The following statements are equivalent:

(i) $\left(x_{n}\right)$ is a d-Cauchy sequence;

(ii) for any $c \gg 0$, there is $k \in \mathbb{N}$ such that

$$
d\left(x_{n}, x_{m}\right) \preceq c \quad \text { for } n, m \geq k ;
$$

(iii) for any subsequence $\left(x_{k_{n}}\right)$ of $\left(x_{n}\right), d\left(x_{n}, x_{k_{n}}\right) \stackrel{K}{\longrightarrow} 0$;

(iv) for any $c \gg 0,\left(x_{n}\right)$ is a $\rho_{c}$-Cauchy sequence;

(v) there exists $c \gg 0$ such that $\left(x_{n}\right)$ is a $\rho_{c}$-Cauchy sequence;

(vi) there exists a sequence $\left(c_{n}\right)$ in $E$ such that $\left\|c_{n}\right\| \rightarrow 0$ and

$$
d\left(x_{i}, x_{j}\right) \preceq c_{n} \quad \text { for } i, j \geq n .
$$


Proof. (i) $\Rightarrow$ (ii) and (ii) $\Rightarrow$ (iii) are obvious. (iii) $\Leftrightarrow($ iv) and (iv) $\Leftrightarrow(v)$ follow from Theorem 2.5, if we invoke the fact that $\left(x_{n}\right)$ is a $\rho_{c}$-Cauchy sequence if and only if $\rho_{c}\left(x_{n}, x_{k_{n}}\right) \rightarrow 0$ for any subsequence $\left(x_{k_{n}}\right)$ of $\left(x_{n}\right)$.

To show (iii) $\Rightarrow$ (i) suppose, on the contrary, that there exist $c \gg 0$ and increasing sequences $\left(k_{n}\right)$ and $\left(m_{n}\right)$ of positive integers such that the condition " $d\left(x_{k_{n}}, x_{m_{n}}\right) \ll c$ " does not hold for any $n \in \mathbb{N}$. By (iii), we have that

$$
d\left(x_{k_{n}}, x_{m_{n}}\right) \preceq d\left(x_{k_{n}}, x_{n}\right)+d\left(x_{n}, x_{m_{n}}\right) \ll c
$$

for sufficiently large $n$, which yields a contradiction.

Finally, (i) $\Leftrightarrow($ vi) can be proved by a similar argument as in the proof of (vi) $\Rightarrow$ (viii) and (viii) $\Rightarrow$ (i) of Theorem 2.5.

Let us notice that Corollary 2.7 also implies that a $K$-metric $d$ and a metric $\rho_{c}$ are equivalent. This is a consequence of the following result, a particular case of which was proved in our paper [18].

Proposition 2.8. Let $d$ and $\rho$ be $K$-metrics or real-valued metrics on $X$. If $d$ and $\rho$ are Cauchy equivalent, then we have the following:

(1) $d$ and $\rho$ are equivalent;

(2) $(X, d)$ is complete if and only if $(X, \rho)$ is complete.

Proof. For example, assume that $d$ is a $K$-metric and $\rho$ is a real-valued metric. Let $\left(x_{n}\right)$ be $d$-convergent to $x \in X$. For $n \in \mathbb{N}$, set

$$
y_{2 n-1}:=x_{n} \text { and } y_{2 n}:=x \text {. }
$$

Then $y_{n} \stackrel{d}{\rightarrow} x$, so by [15, Lemma 3$],\left(y_{n}\right)$ is a $d$-Cauchy sequence. By hypothesis, $\left(y_{n}\right)$ is a $\rho$-Cauchy sequence, so in particular, $\rho\left(y_{2 n-1}, y_{2 n}\right) \rightarrow 0$, which means that $\left(x_{n}\right)$ is $\rho$-convergent to $x$. The same argument shows that $\rho$-convergence implies $d$-convergence. Now statement (2) is obvious.

\section{Cantor's intersection theorem for $K$-metric spaces}

In a $K$-metric space $(X, d)$ it is natural to consider the following two notions of boundedness of a set. We say that $A \subseteq X$ is order-bounded if there exists $c \in K$ such that $d(x, y) \preceq c$ for $x, y \in A$. $A$ is called norm-bounded if its norm-diameter

$$
\operatorname{diam} A:=\sup _{x, y \in A}\|d(x, y)\|
$$

is finite. In general, the two notions do not coincide. However, some connections between them can be established under additional assumptions on a cone $K$ as will be shown in what follows.

Let us notice that if $A$ is order-bounded and a cone $K$ is strongly minihedral, then the order-diameter of $A$ can be defined as

$$
\operatorname{diam}_{\preceq} A:=\sup \{d(x, y): x, y \in A\} .
$$


In this case, Cantor's intersection theorem for $K$-metric spaces can be formulated as its classical version. Recently, this has been done by Alnafei, Radenović and Shahzad [2] in the following form.

Theorem 3.1. Let $(X, d)$ be a K-metric space over a solid and strongly minihedral cone $K$. Then $(X, d)$ is complete if and only if any decreasing sequence $\left(A_{n}\right)$ of nonempty closed and order-bounded subsets of $X$, with

$$
\left\|\operatorname{diam}_{\preceq} A_{n}\right\| \rightarrow 0,
$$

has a nonempty intersection.

However, in general, it is not possible to attribute the order-diameter to any order-bounded set as shown in the following example.

Example 3.2. Let $E:=C([0,2])$ be endowed with the max-norm and let $K$ be the positive cone in $E$, i.e., $K:=\{f \in E: f \geq 0\}$. It is known that $K$ is not strongly minihedral (cf. [14, Example 1.3.1, p. 12]). Set $X:=E$ and for $f, g \in X, d(f, g):=|f-g|$. It is easily seen that $d$ is a $K$-metric. Set

$$
\begin{aligned}
A:=\{f \in C([0,2]): 0 \leq f(t) \leq 1 \text { for } t \in[0,1] \\
\\
\text { and } 0 \leq f(t) \leq 2 \text { for } t \in(1,2]\} .
\end{aligned}
$$

Then $A \subseteq X$ and $d(f, g) \preceq h_{0}$ for $f, g \in A$, where $h_{0}(t):=2$ for $t \in[0,2]$, so $A$ is order-bounded. It is easily seen that $\{d(f, g): f, g \in A\}=A$. An elementary argument shows that $\sup A$ does not exist. Thus the set

$$
\{d(f, g): f, g \in A\}
$$

has no supremum.

Fortunately, Cantor's theorem can be formulated without referring to diameters of sets. We omit an obvious proof of the following proposition.

Proposition 3.3. Let $(X, \rho)$ be a metric space and let $\left(A_{n}\right)$ be a sequence of bounded subsets of $X$. Then $\operatorname{diam} A_{n} \rightarrow 0$ if and only if there exists a sequence $\left(\alpha_{n}\right)$ of reals such that $\alpha_{n} \rightarrow 0$ and $\rho(x, y) \leq \alpha_{n}$ for $x, y \in A_{n}$ and $n \in \mathbb{N}$.

In a $K$-metric setting we may consider the following two versions of the latter condition of Proposition 3.3, which, however, turn out to be equivalent.

Proposition 3.4. Let $(X, d)$ be a $K$-metric space over a solid cone $K$ in a Banach space $E$, and let $\left(A_{n}\right)$ be a sequence of subsets of $X$. The following statements are equivalent:

(i) there exists a sequence $\left(c_{n}\right)$ in $E$ such that $c_{n} \stackrel{K}{\longrightarrow} 0$ and $d(x, y) \preceq c_{n}$ for $x, y \in A_{n}$ and $n \in \mathbb{N}$;

(ii) there exists a sequence $\left(c_{n}\right)$ in $E$ such that $\left\|c_{n}\right\| \rightarrow 0$ and $d(x, y) \preceq c_{n}$ for $x, y \in A_{n}$ and $n \in \mathbb{N}$.

Proof. (i) $\Rightarrow\left(\right.$ ii): Assume that $\left(c_{n}\right)$ is as in (i). By Theorem $2.5((\mathrm{i}) \Rightarrow($ viii)), there exists a sequence $\left(d_{n}\right)$ such that $\left\|d_{n}\right\| \rightarrow 0$ and $c_{n} \preceq d_{n}$. Obviously, $d(x, y) \preceq d_{n}$ for $x, y \in A_{n}$ and $n \in \mathbb{N}$, so (ii) holds. 
(ii) $\Rightarrow(\mathrm{i})$ : Now let $\left(c_{n}\right)$ be as in (ii). By Theorem $2.5(($ viii $) \Rightarrow(\mathrm{i}))$, with $a_{n}:=c_{n}$ and $a:=0$, we get that

$$
c_{n} \stackrel{K}{\longrightarrow} 0,
$$

so (i) holds.

It turns out that the convergence of norm-diameters of sets to 0 is a stronger assumption than (i) of Proposition 3.4.

Proposition 3.5. Let $(X, d)$ be a $K$-metric space over a solid cone $K$ in $X$. Then we have the following.

(1) If a set $A \subseteq X$ is norm-bounded, then it is order-bounded.

(2) If $\left(A_{n}\right)$ is a sequence of subsets of $X$ and

$$
\operatorname{diam} A_{n}=\sup _{x, y \in A_{n}}\|d(x, y)\| \rightarrow 0,
$$

then there exists a sequence $\left(c_{n}\right)$ in $E$ such that $\left\|c_{n}\right\| \rightarrow 0$ and $d(x, y) \preceq$ $c_{n}$ for $x, y \in A_{n}$ and $n \in \mathbb{N}$.

Proof. Let $A \subseteq X$ and $\operatorname{diam} A<\infty$. Fix $c \gg 0$. Then for some $\alpha>0$, the closed ball $B(c, \alpha)$ is contained in $K$. Hence for any $\lambda>0$,

$$
\lambda B(c, \alpha)=B(\lambda c, \lambda \alpha) \subseteq K .
$$

In particular, for $\lambda:=\operatorname{diam} A / \alpha$ we get that

$$
B\left(\frac{\operatorname{diam} A}{\alpha} c, \operatorname{diam} A\right) \subseteq K .
$$

Hence, if $a \in E$ and $\|a\| \leq \operatorname{diam} A$, then

$$
\frac{\operatorname{diam} A}{\alpha} c-a \in K,
$$

i.e., $a \preceq(\operatorname{diam} A / \alpha) c$. This yields

$$
d(x, y) \preceq \frac{\operatorname{diam} A}{\alpha} c \quad \text { for } x, y \in A .
$$

Now assume that $A_{n} \subseteq X$ for $n \in \mathbb{N}$ and $\operatorname{diam} A_{n} \rightarrow 0$. If $c \gg 0$ and $\alpha$ is as above, then by the proof of point (1), we have that $d(x, y) \preceq c_{n}$ for $x, y \in A_{n}$ and $n \in \mathbb{N}$, where $c_{n}:=\left(\operatorname{diam} A_{n} / \alpha\right) c$. Clearly, $\left\|c_{n}\right\| \rightarrow 0$ which completes the proof.

The following example shows that in general the converse of Proposition $3.5(2)$ is false.

Example 3.6. Let $E:=C^{1}([0,1])$ be endowed with the norm

$$
\|f\|:=\max _{t \in[0,1]}|f(t)|+\max _{t \in[0,1]}\left|f^{\prime}(t)\right| \quad \text { for } f \in E .
$$

Let $K$ be the positive cone in $E, X:=K$ and for $f, g \in X$,

$$
d(f, g):= \begin{cases}f+g & \text { if } f \neq g, \\ 0 & \text { if } f=g .\end{cases}
$$


For $n \in \mathbb{N}$, set

$$
A_{n}:=\left\{f \in X: d(f, 0) \preceq c_{n}\right\},
$$

where $c_{n}(t):=t^{n} / n$ for $t \in[0,1]$. Then $0 \in A_{n}$ and $c_{n} \in A_{n}$, so

$$
\operatorname{diam} A_{n} \geq\left\|d\left(0, c_{n}\right)\right\|=\left\|c_{n}\right\|=\frac{1}{n}+1
$$

and hence diam $A_{n} \nrightarrow 0$. On the other hand, for $f, g \in A_{n}, d(f, g) \preceq 2 c_{n} \preceq d_{n}$, where $d_{n}(t):=2 / n$ for $t \in[0,1]$. Obviously, $\left\|d_{n}\right\| \rightarrow 0$.

Observe that the cone $K$ in Example 3.6 is not normal. In fact, it turns out that for any nonnormal cone $K$, there exist a $K$-metric space and a sequence of its subsets with the properties as in Example 3.6. Namely, we have the following characterization of normal cones.

Proposition 3.7. Let $K$ be an arbitrary cone in a Banach space $E$. The following statements are equivalent:

(i) $K$ is normal;

(ii) for any $K$-metric space $(X, d)$ and any $A \subseteq X$, if $A$ is order-bounded, then it is norm-bounded;

(iii) for any $K$-metric space $(X, d)$ and any decreasing sequence $\left(A_{n}\right)$ of subsets of $X$, if there exists a sequence $\left(c_{n}\right)$ in $E$ such that $\left\|c_{n}\right\| \rightarrow 0$ and $d(x, y) \preceq c_{n}$ for $x, y \in A_{n}$ and $n \in \mathbb{N}$, then $\operatorname{diam} A_{n} \rightarrow 0$.

Proof. (i) $\Rightarrow$ (ii) and (i) $\Rightarrow$ (iii) follow from the fact that $K$ is normal if and only if the norm on $E$ is semimonotone (cf. [1, Theorem 2.38]), i.e., there is $\gamma>0$ such that for $a, b \in E, 0 \preceq a \preceq b$ implies $\|a\| \leq \gamma\|b\|$. Hence, if $\left(A_{n}\right)$ is as in (iii), then $\|d(x, y)\| \leq \gamma\left\|c_{n}\right\|$ for $x, y \in A_{n}$ and $n \in \mathbb{N}$, so $\operatorname{diam} A_{n} \rightarrow 0$.

Now we show simultaneously implications (ii) $\Rightarrow$ (i) and (iii) $\Rightarrow$ (i). Our proof is partially inspired by the proof of [1, Theorem 2.40]. Suppose, on the contrary, that $K$ is not normal. Then there exist sequences $\left(a_{n}\right)$ and $\left(b_{n}\right)$ in $E$ such that for $n \in \mathbb{N}$,

$$
0 \preceq a_{n} \preceq b_{n} \quad \text { and } \quad\left\|a_{n}\right\|>n^{3}\left\|b_{n}\right\| .
$$

Hence $\left\|a_{n}\right\|>0$, so $a_{n} \neq 0$, which yields $b_{n} \neq 0$. Thus we may set for $n \in \mathbb{N}$,

$$
a_{n}^{\prime}:=\frac{1}{n^{2}\left\|b_{n}\right\|} a_{n} \quad \text { and } \quad b_{n}^{\prime}:=\frac{1}{n^{2}\left\|b_{n}\right\|} b_{n} .
$$

Then $0 \preceq a_{n}^{\prime} \preceq b_{n}^{\prime}$. The series $\sum_{n=1}^{\infty} b_{n}^{\prime}$ is absolutely convergent in a Banach space $E$, so we may define

$$
b_{n}^{\prime \prime}:=\sum_{i=n}^{\infty} b_{i}^{\prime} \quad \text { for } n \in \mathbb{N} .
$$

Then $\left\|b_{n}^{\prime \prime}\right\| \rightarrow 0$ and $b_{n}^{\prime \prime} \in K$ since $K$ is closed. Now set $X:=K$ and for $x, y \in X$,

$$
d(x, y):= \begin{cases}x+y & \text { if } x \neq y \\ 0 & \text { if } x=y\end{cases}
$$


Then $d$ is a $K$-metric on $X$. For $n \in \mathbb{N}$, define

$$
A_{n}:=\left\{a_{k}^{\prime}: k \geq n\right\} \cup\{0\} .
$$

Clearly, $\left(A_{n}\right)$ is decreasing and for any $k \geq n$,

$$
\operatorname{diam} A_{n} \geq\left\|d\left(a_{k}^{\prime}, 0\right)\right\|=\left\|a_{k}^{\prime}\right\|=\frac{\left\|a_{k}\right\|}{k^{2}\left\|b_{k}\right\|}>k,
$$

so $\operatorname{diam} A_{n}=\infty$.

On the other hand, if $x, y \in A_{n}$, then either $d(x, y)=0, d(x, y)=a_{k}^{\prime}$, or $d(x, y)=a_{k}^{\prime}+a_{m}^{\prime}$ for some $k, m \geq n$ with $k \neq m$. In each case,

$$
d(x, y) \preceq b_{k}^{\prime}+b_{m}^{\prime} \preceq b_{n}^{\prime \prime},
$$

which contradicts (iii) since $\left\|b_{n}^{\prime \prime}\right\| \rightarrow 0$ and $\operatorname{diam} A_{n} \nrightarrow 0$. Moreover, each $A_{n}$ is order-bounded and is not norm-bounded, which in turn contradicts (ii).

Given $x \in X$ and $a \in K$, we define the closed ball

$$
B(x, a):=\{y \in X: d(x, y) \preceq a\} .
$$

The following two results will be used in the proof of Cantor's theorem.

Proposition 3.8. Let $(X, d)$ be a $K$-metric space over a solid cone $K$. Then every ball $B(x, a)$ is d-closed.

Proof. Let $y_{n} \in B(x, a)$ for $n \in \mathbb{N}$ and $y_{n} \stackrel{d}{\rightarrow} y$. Then

$$
d(x, y) \preceq d\left(x, y_{n}\right)+d\left(y_{n}, y\right) \preceq a+d\left(y_{n}, y\right) .
$$

Fix $c \gg 0$. By Theorem $2.5((\mathrm{i}) \Rightarrow(\mathrm{v})), d\left(y_{n}, y\right) \stackrel{K}{\longrightarrow} 0$ implies that

$$
\left\|d\left(y_{n}, y\right)\right\|_{c} \rightarrow 0 \text {. }
$$

Since by Corollary $2.2, K$ is $\|\cdot\|_{c}$-closed, (3.1) yields that $d(x, y) \preceq a$, i.e., $y \in B(x, a)$.

Proposition 3.9. Let $(X, d)$ be a $K$-metric space over a solid cone $K$ and let $\left(x_{n}\right)$ be a d-Cauchy sequence in $X$. Then $\left(x_{n}\right)$ is d-convergent if and only if it contains a d-convergent subsequence.

Proof. The part "only if" is trivial. So assume that $x_{k_{n}} \stackrel{d}{\longrightarrow} x$. Then

$$
0 \preceq d\left(x_{n}, x\right) \preceq d\left(x_{n}, x_{k_{n}}\right)+d\left(x_{k_{n}}, x\right) .
$$

Fix $c \gg 0$. By Theorem $2.5((\mathrm{i}) \Rightarrow(\mathrm{v}))$,

$$
d\left(x_{n}, x_{k_{n}}\right) \stackrel{K}{\longrightarrow} 0 \text { and } d\left(x_{k_{n}}, x\right) \stackrel{K}{\longrightarrow} 0
$$

imply that

$$
\left\|d\left(x_{n}, x_{k_{n}}\right)+d\left(x_{k_{n}}, x\right)\right\|_{c} \rightarrow 0 .
$$

By Corollary 2.2, $K$ is $\|\cdot\|_{c}$-normal, so (3.2) implies that $\left\|d\left(x_{n}, x\right)\right\|_{c} \rightarrow 0$. Now Corollary $2.6(($ vii $) \Rightarrow(\mathrm{i}))$ yields that

$$
x_{n} \stackrel{d}{\longrightarrow} x .
$$

The following is Cantor's intersection theorem for $K$-metric spaces. 
Theorem 3.10. Let $(X, d)$ be a $K$-metric space over a solid cone $K$ in a Banach space $E$. The following statements are equivalent:

(i) $(X, d)$ is complete;

(ii) every decreasing sequence $\left(A_{n}\right)$ of nonempty closed subsets of $X$ such that there exists a sequence $\left(c_{n}\right)$ in $E$ with

$$
c_{n} \stackrel{K}{\longrightarrow} 0 \text { and } \quad d(x, y) \preceq c_{n} \quad \text { for } x, y \in A_{n} \text { and } n \in \mathbb{N},
$$

has a nonempty intersection and $\bigcap_{n \in \mathbb{N}} A_{n}$ is a singleton;

(iii) every decreasing sequence $\left(A_{n}\right)$ of nonempty closed subsets of $X$ such that there exists a sequence $\left(c_{n}\right)$ in $E$ with $\left\|c_{n}\right\| \rightarrow 0$ and $d(x, y) \preceq c_{n}$ for $x, y \in A_{n}$ and $n \in \mathbb{N}$, has a nonempty intersection and $\bigcap_{n \in \mathbb{N}} A_{n}$ is a singleton;

(iv) every decreasing sequence $\left(B\left(x_{n}, a_{n}\right)\right)$ of closed balls in $X$ with $a_{n} \gg 0$ and $\left\|a_{n}\right\| \rightarrow 0$ has a nonempty intersection.

Proof. (i) $\Rightarrow$ (ii): Let a sequence $\left(A_{n}\right)$ be as in (ii). By Theorem $2.5((\mathrm{i}) \Rightarrow($ viii)), there exists a sequence $\left(d_{n}\right)$ in $E$ such that $\left\|d_{n}\right\| \rightarrow 0$ and $c_{n} \preceq d_{n}$. Then we have

$$
d(x, y) \preceq d_{n} \quad \text { for } x, y \in A_{n} .
$$

Fix $c \gg 0$. By Corollary 2.2, $\|\cdot\|_{c}$ is monotone and $\|\cdot\|$-continuous, so (3.3) yields that

$$
\|d(x, y)\|_{c} \leq\left\|d_{n}\right\|_{c} \quad \text { for } x, y \in A_{n},
$$

which implies that the diameters of sets $A_{n}$ with respect to the metric $\rho_{c}$ defined by $(2.2)$ converge to 0 . Since by Corollary 2.7, $d$ and $\rho_{c}$ are Cauchy equivalent, we may conclude using Proposition 2.8 that each $A_{n}$ is $\rho_{c}$-closed and $\left(X, \rho_{c}\right)$ is complete. Thus, by the classical Cantor's intersection theorem, $\bigcap_{n \in \mathbb{N}} A_{n}$ is a singleton.

The equivalence (ii) $\Leftrightarrow$ (iii) follows from Proposition 3.4.

(iii) $\Rightarrow$ (iv): Let a sequence $\left(B\left(x_{n}, a_{n}\right)\right)$ be as in (iv). By Proposition 3.8, each $B\left(x_{n}, a_{n}\right)$ is $d$-closed. Moreover, for $x, y \in B\left(x_{n}, a_{n}\right)$,

$$
d(x, y) \preceq d\left(x, x_{n}\right)+d\left(x_{n}, y\right) \preceq 2 a_{n} .
$$

Since $\left\|a_{n}\right\| \rightarrow 0$, (iii) yields that $\bigcap_{n \in \mathbb{N}} B\left(x_{n}, a_{n}\right)$ is nonempty.

(iv) $\Rightarrow(\mathrm{i})$ : Here we use a similar argument as in the classical proof of Cantor's theorem. Let $\left(x_{n}\right)$ be a Cauchy sequence in $(X, d)$. Fix $c \gg 0$. Then there exists a subsequence $\left(x_{k_{n}}\right)$ such that

$$
d\left(x_{k_{n}}, x_{k_{n+1}}\right) \preceq \frac{c}{2^{n+1}} .
$$

Set $B_{n}:=B\left(x_{k_{n}}, c / 2^{n}\right)$ for $n \in \mathbb{N}$. If $x \in B_{n+1}$, then

$$
d\left(x, x_{k_{n}}\right) \preceq d\left(x, x_{k_{n+1}}\right)+d\left(x_{k_{n+1}}, x_{k_{n}}\right) \preceq \frac{c}{2^{n+1}}+\frac{c}{2^{n+1}}=\frac{c}{2^{n}},
$$

so $x \in B_{n}$, and hence $B_{n+1} \subseteq B_{n}$. Thus (iv) yields the existence of $x_{0}$ in $\bigcap_{n \in \mathbb{N}} B_{n}$. Then

$$
d\left(x_{0}, x_{k_{n}}\right) \preceq \frac{c}{2^{n}},
$$


so by Corollary 2.6,

$$
x_{k_{n}} \stackrel{d}{\longrightarrow} x_{0} .
$$

Now Proposition 3.9 ensures that also $\left(x_{n}\right)$ converges to $x_{0}$. Consequently, $(X, d)$ is complete.

\section{A contraction principle for $K$-metric spaces via Cantor's theorem}

Janković, Kadelburg and Radenović [19] have observed that a number of fixed point theorems for mappings on $K$-metric spaces can be derived from the corresponding results in metric spaces with the help of the following Krein's characterization of normal cones (cf. [1, Theorem 2.38]).

Theorem 4.1 (Krein). A cone $K$ in a Banach space $E$ is normal if and only if $E$ admits an equivalent monotone norm.

Following their approach, we present here a proof of the Huang-Zhang theorem [15] via the classical contraction principle. A similar argument can be used in proofs of many other fixed point results mentioned in [19].

Theorem 4.2 (Huang-Zhang). Let $(X, d)$ be a complete $K$-metric space over a solid normal cone $K$ in a Banach space $E$. Let a mapping $T: X \rightarrow X$ be such that for some $\lambda \in[0,1)$,

$$
d(T x, T y) \preceq \lambda d(x, y) \quad \text { for } x, y \in X .
$$

Then $T$ has a unique fixed point $x_{*}$ and for any $x_{0} \in X, T^{n} x_{0} \stackrel{d}{\rightarrow} x_{*}$.

Proof. Let $\|\cdot\|$ be an equivalent monotone norm on $E$. Set

$$
\rho(x, y):=\|d(x, y)\| \text { for } x, y \in X .
$$

Then $\rho$ is a metric on $X$ and it can easily be shown, using the monotonicity of the norm, that for any $\left(x_{n}\right)$ in $X,\left(x_{n}\right)$ is a $d$-Cauchy sequence if and only if $\left\|d\left(x_{n}, x_{m}\right)\right\| \rightarrow 0$ as $n, m \rightarrow \infty$, i.e., $d$ and $\rho$ are Cauchy equivalent. Hence, by Proposition 2.8, $d$ and $\rho$ are equivalent, and $(X, \rho)$ is complete. Moreover, the monotonicity of $\|\cdot\|$ implies that for $x, y \in X$,

$$
\|d(T x, T y)\| \leq\|\lambda d(x, y)\|=\lambda\|d(x, y)\|,
$$

i.e., $T$ is a $\rho$-contraction. Now it suffices to apply the classical contraction principle.

Rezapour and Hamlbarani [26] have obtained an extension of Theorem 4.2 by omitting the assumption of normality of $K$. Then, however, as observed by Kadelburg, Radenović and Rakočević [20], it is possible to repeat the above proof only replacing the norm $\|\cdot\|$ by the Minkowski functional $\|\cdot\|_{c}$ defined by (2.1), where $c \gg 0$. So also this result is subsumed by the classical Banach's fixed point theorem. (Let us also notice that interesting applications of the Minkowski functional technique to Caristi-type fixed point results were found recently by Khamsi and Wojciechowski [21].) 
However, the problem is more delicate if we consider a mapping $T$ satisfying condition (1.3), i.e.,

$$
d(T x, T y) \preceq \Lambda(d(x, y)) \quad \text { for } x, y \in X,
$$

where $\Lambda$ is a linear continuous positive operator on $E$ with the spectral radius less than 1. If $K$ is normal and $\rho$ is a metric from the proof of Theorem 4.2, then we obtain only that

$$
\rho(T x, T y) \leq\|\Lambda\| \rho(x, y) .
$$

In general, $\|\Lambda\| \geq 1$, but since $r(\Lambda)<1$, there exists $p \in \mathbb{N}$ such that $\left\|\Lambda^{p}\right\|<1$. Then, by monotonicity of $\Lambda$, we may infer that $T^{p}$ is a $\rho$-contraction. Since $T$ is continuous, $\left[17\right.$, Theorem 2.1] implies that there exists a complete metric $\rho^{\prime}$ equivalent to $\rho$ such that $T$ is a $\rho^{\prime}$-contraction, so the classical contraction principle is applicable. In fact, this argument can be extended to mappings satisfying (1.3) with a nonlinear operator $\Lambda$ (see [18, Theorem 6]). Another argument (in the linear case) is given in [6].

It seems that for nonnormal cones the problem is yet more difficult. In this case, using a metric $\rho$ induced on $X$ by the norm $\|\cdot\|_{c}$, we get from (1.3) by monotonicity of $\|\cdot\|_{c}$ that for any $n \in \mathbb{N}$,

$$
\rho\left(T^{n} x, T^{n} y\right) \leq\left\|\Lambda^{n}(d(x, y))\right\|_{c} \leq\left\|\Lambda^{n}\right\|_{c} \rho(x, y)
$$

(it can be shown that $\Lambda$ is $\|\cdot\|_{c}$-continuous), but now it is not clear if the spectral radius of $\Lambda$ with respect to $\|\cdot\|_{c}$ is less than 1 since by [1, Theorem 2.63], the norms $\|\cdot\|_{c}$ and $\|\cdot\|$ are not equivalent and, moreover, $\left(E,\|\cdot\|_{c}\right)$ is not a Banach space. Here we show that a fixed point theorem for mappings on a $K$-metric space over a solid (not necessarily normal) cone can be derived from our Cantor's intersection theorem. The result is a special case of the Cvetković-Rakočević theorem [4], in which a mapping $T$ is such that for any $x, y \in X$, there exists $u \in\{d(x, y), d(x, T x), d(y, T y), d(x, T y), d(y, T x)\}$ with $d(T x, T y) \preceq \Lambda(u)$.

Theorem 4.3. Let $(X, d)$ be a complete $K$-metric space over a solid cone $K$ in a Banach space $E$. Let $T: X \rightarrow X$ be a mapping such that

$$
d(T x, T y) \preceq \Lambda(d(x, y)) \quad \text { for } x, y \in X,
$$

where $\Lambda: E \rightarrow E$ is a linear positive operator with the spectral radius $r(\Lambda)$ less than 1 . Then $T$ has a unique fixed point $x_{*}$ and for any $x_{0} \in X$,

$$
T^{n} x_{0} \stackrel{d}{\longrightarrow} x_{*} .
$$

The proof of Theorem 4.3 will be preceded by some auxiliary results. Also, let us notice that an operator $\Lambda$ as in Theorem 4.3 is automatically continuous, so $r(\Lambda)$ is well defined. Indeed, by [5, Proposition 19.1], every solid cone $K$ is generating, i.e., $K-K=E$, so by [1, Theorem 2.32], every linear positive operator from $E$ to $E$ is then continuous.

Janković, Kadelburg and Radenović [19, Example 6.1] have presented that a $K$-metric $d$ need not be continuous (even with respect to one variable): 
it may happen that

$$
x_{n} \stackrel{d}{\longrightarrow} x,
$$

but $\left\|d\left(x_{n}, x\right)\right\| \nrightarrow 0$. However, $d$ is continuous in other sense according to the following proposition.

Proposition 4.4. Let $(X, d)$ be a $K$-metric space over a solid cone $K, x, y \in X$ and let $\left(x_{n}\right),\left(y_{n}\right)$ be sequences in $X$. If

$$
x_{n} \stackrel{d}{\longrightarrow} x \text { and } \quad y_{n} \stackrel{d}{\longrightarrow} y,
$$

then

$$
d\left(x_{n}, y_{n}\right) \stackrel{K}{\longrightarrow} d(x, y) .
$$

Proof. By Corollary $2.6((\mathrm{i}) \Rightarrow($ viii $))$, there exist sequences $\left(c_{n}\right)$ and $\left(d_{n}\right)$ in $E$ such that

$$
\left\|c_{n}\right\| \rightarrow 0, \quad\left\|d_{n}\right\| \rightarrow 0, \quad d\left(x_{n}, x\right) \preceq c_{n}, \quad d\left(y_{n}, y\right) \preceq d_{n} .
$$

Hence, by the triangle inequality, we get

$$
d\left(x_{n}, y_{n}\right)-d(x, y) \preceq d\left(x_{n}, x\right)+d\left(y_{n}, y\right) \preceq c_{n}+d_{n} .
$$

Interchanging $x_{n}$ with $x$ and $y_{n}$ with $y$, and multiplying by -1 yields

$$
-c_{n}-d_{n} \preceq-d\left(x_{n}, x\right)-d\left(y_{n}, y\right) \preceq d\left(x_{n}, y_{n}\right)-d(x, y) .
$$

Thus we obtain that

$$
-\left(c_{n}+d_{n}\right) \preceq d\left(x_{n}, y_{n}\right)-d(x, y) \preceq c_{n}+d_{n} .
$$

Since $\left\|c_{n}+d_{n}\right\| \rightarrow 0$, Theorem $2.5(($ viii $) \Rightarrow(\mathrm{i}))$ implies that

$$
d\left(x_{n}, y_{n}\right) \stackrel{K}{\longrightarrow} d(x, y) .
$$

Lemma 4.5. Let $T$ be as in Theorem 4.3. Then $T$ is d-continuous.

Proof. Let

$$
x_{n} \stackrel{d}{\longrightarrow} x .
$$

By Corollary $2.6((\mathrm{i}) \Rightarrow($ viii $))$, there exists a sequence $\left(c_{n}\right)$ such that $\left\|c_{n}\right\| \rightarrow 0$ and $d\left(x_{n}, x\right) \preceq c_{n}$. By hypothesis, $\Lambda$ is positive, so is monotone. Hence we get

$$
d\left(T x_{n}, T x\right) \preceq \Lambda\left(d\left(x_{n}, x\right)\right) \preceq \Lambda\left(c_{n}\right) .
$$

Since $\Lambda$ is $\|\cdot\|$-continuous, we have that $\left\|\Lambda\left(c_{n}\right)\right\| \rightarrow 0$, so by Corollary 2.6 $(($ viii $) \Rightarrow(\mathrm{i}))$,

$$
T x_{n} \stackrel{d}{\longrightarrow} T x .
$$

Lemma 4.6. Let $T$ be as in Theorem 4.3 and $c \gg 0$. Set

$$
A:=\{x \in X: d(x, T x) \preceq c\} .
$$

Then $A$ is nonempty and closed, and for any $x, y \in A$,

$$
d(x, y) \preceq 2(I-\Lambda)^{-1}(c) .
$$


Proof. We show that $A$ is nonempty. Fix $x_{0} \in X$. By monotonicity of $\Lambda$,

$$
d\left(T^{n} x_{0}, T^{n+1} x_{0}\right) \preceq \Lambda^{n}\left(d\left(x_{0}, T x_{0}\right)\right) \quad \text { for } n \in \mathbb{N} .
$$

Since $r(\Lambda)<1$, we have that

$$
\left\|\Lambda^{n}\left(d\left(x_{0}, T x_{0}\right)\right)\right\| \rightarrow 0 .
$$

Thus by Theorem $2.5(($ viii $) \Rightarrow(\mathrm{i}))$, we get that

$$
d\left(T^{n} x_{0}, T^{n+1} x_{0}\right) \stackrel{K}{\longrightarrow} 0 .
$$

Hence there is $p \in \mathbb{N}$ such that $T^{p} x_{0} \in A$.

We show that $A$ is closed. Set $\varphi(x):=d(x, T x)$ for $x \in X$. By Proposition 4.4 and Lemma 4.5, $\varphi$ is continuous in the sense that if

$$
x_{n} \stackrel{d}{\longrightarrow} x,
$$

then

$$
\varphi\left(x_{n}\right) \stackrel{K}{\longrightarrow} \varphi(x) .
$$

By Theorem 2.5, the latter convergence is equivalent to the convergence

$$
\left\|\varphi\left(x_{n}\right)-\varphi(x)\right\|_{c} \rightarrow 0 .
$$

The continuity of $\varphi$ implies the closeness of $A$ since $A=\varphi^{-1}([-c, c])$ and by Corollary $2.2,[-c, c]$ is $\|\cdot\|_{c}$-closed.

Finally, fix $x, y \in A$. Then

$$
d(x, y) \preceq d(x, T x)+d(T x, T y)+d(y, T y) \preceq 2 c+\Lambda(d(x, y))
$$

and hence

$$
(I-\Lambda)(d(x, y)) \preceq 2 c .
$$

Since $r(\Lambda)<1$, the operator $I-\Lambda$ is invertible and $(I-\Lambda)^{-1}$ can be expressed by the Neumann series:

$$
(I-\Lambda)^{-1}=\sum_{n=0}^{\infty} \Lambda^{n}
$$

Since $\Lambda$ is increasing, so is each iterate $\Lambda^{n}$. Hence, given $a, b \in E$ with $a \preceq b$, we may infer that

$$
\sum_{k=0}^{n} \Lambda^{k} a \preceq \sum_{k=0}^{n} \Lambda^{k} b .
$$

By closeness of $K$, letting $n$ tend to $\infty$, we get

$$
\sum_{k=0}^{\infty} \Lambda^{k} a \preceq \sum_{k=0}^{\infty} \Lambda^{k} b,
$$

i.e., $(I-\Lambda)^{-1} a \preceq(I-\Lambda)^{-1} b$, which means that $(I-\Lambda)^{-1}$ is increasing. Hence, by (4.2), we obtain that (4.1) holds. 
Proof of Theorem 4.3. Fix $c \gg 0$ and for $n \in \mathbb{N}$, set

$$
A_{n}:=\left\{x \in X: d(x, T x) \preceq \frac{1}{n} c\right\} .
$$

Clearly, $\left(A_{n}\right)$ is decreasing. For any $n \in \mathbb{N},(1 / n) c \gg 0$, so by Lemma 4.6, each $A_{n}$ is nonempty and closed, and

$$
d(x, y) \preceq \frac{2}{n}(I-\Lambda)^{-1}(c) \quad \text { for } x, y \in A_{n} .
$$

Set $c_{n}:=(2 / n)(I-\Lambda)^{-1}(c)$. Clearly, $\left\|c_{n}\right\| \rightarrow 0$, so Theorem 3.10 implies that

$$
\bigcap_{n \in \mathbb{N}} A_{n}=\left\{x_{*}\right\} \quad \text { for some } x_{*} \in X \text {. }
$$

By closeness of $K$, we easily get that $\operatorname{Fix} T=\bigcap_{n \in \mathbb{N}} A_{n}$, so $x_{*}$ is the unique fixed point of $T$.

Now fix $x_{0} \in X$. For any $n \in \mathbb{N}$, we have

$$
d\left(T^{n} x_{0}, x_{*}\right)=d\left(T^{n} x_{0}, T^{n} x_{*}\right) \preceq \Lambda^{n}\left(d\left(x_{0}, x_{*}\right)\right) .
$$

Since $\left\|\Lambda^{n}\left(d\left(x_{0}, x_{*}\right)\right)\right\| \rightarrow 0$, Corollary 2.6 yields that

$$
T^{n} x_{0} \stackrel{d}{\longrightarrow} x_{*} \text {. }
$$

Finally, let us notice that it is also possible to give another proof of Theorem 4.3 by modifying the classical proof of the contraction principle.

\section{Acknowledgment}

We are grateful to the referee for calling our attention to the papers $[4,21]$.

\section{References}

[1] C. D. Aliprantis and R. Tourky, Cones and Duality. Grad. Stud. Math. 84, Amer. Math. Soc., Providence, RI, 2007.

[2] S. H. Alnafei, S. Radenović and N. Shahzad, Fixed point theorems for mappings with convex diminishing diameters on cone metric spaces. Appl. Math. Lett. 24 (2011), 2162-2166.

[3] D. W. Boyd and J. S. W. Wong, On nonlinear contractions. Proc. Amer. Math. Soc. 20 (1969), 458-464.

[4] M. Cvetković and Rakočević, Quasi-contraction of Perov type. Appl. Math. Comput. 237 (2014), 712-722.

[5] K. Deimling, Nonlinear Functional Analysis. Springer-Verlag, Berlin, 1985.

[6] E. De Pascale and L. De Pascale, Fixed points for some non-obviously contractive operators. Proc. Amer. Math. Soc. 130 (2002), 3249-3254.

[7] W.-S. Du, A note on cone metric fixed point theory and its equivalence. Nonlinear Anal. 72 (2010), 2259-2261.

[8] J. Dugundji, Positive definite functions and coincidences. Fund. Math. 90 (1975/76), 131-142. 
[9] K. Goebel, An elementary proof of the fixed-point theorem of Browder and Kirk. Michigan Math. J. 16 (1969), 381-383.

[10] K. Goebel and W. A. Kirk, Topics in Metric Fixed Point Theory. Cambridge Stud. Adv. Math. 28, Cambridge University Press, Cambridge, 1990.

[11] K. Goebel and S. Reich, Uniform Convexity, Hyperbolic Geometry, and Nonexpansive Mappings. Monographs and Textbooks in Pure and Applied Mathematics 83, Marcel Dekker, New York, 1984.

[12] A. Granas and J. Dugundji, Fixed Point Theory. Springer Monographs in Mathematics, Springer-Verlag, New York, 2003.

[13] A. Granas and C. D. Horvath, On the order-theoretic Cantor theorem. Taiwanese J. Math. 4 (2000), 203-213.

[14] D. Guo and V. Lakshmikantham, Nonlinear Problems in Abstract Cones. Notes and Reports in Mathematics in Science and Engineering 5, Academic Press, Boston, MA, 1988.

[15] L.-G. Huang and X. Zhang, Cone metric spaces and fixed point theorems of contractive mappings. J. Math. Anal. Appl. 332 (2007), 1468-1476.

[16] J. Jachymski, Around Browder's fixed point theorem for contractions. J. Fixed Point Theory Appl. 5 (2009), 47-61.

[17] J. Jachymski, Remetrization theorems for finite families of mappings and hyperbolic iterated function systems. In: Infinite Products of Operators and Their Applications, Contemp. Math. 636, Amer. Math. Soc., Providence, RI, 2015, 131-139.

[18] J. Jachymski and J. Klima, Around Perov's fixed point theorem for mappings on generalized metric spaces. Fixed Point Theory, to appear.

[19] S. Janković, Z. Kadelburg and S. Radenović, On cone metric spaces: A survey. Nonlinear Anal. 74 (2011), 2591-2601.

[20] Z. Kadelburg, S. Radenović and V. Rakočević, A note on the equivalence of some metric and cone metric fixed point results. Appl. Math. Lett. 24 (2011), $370-374$.

[21] M. A. Khamsi and P. J. Wojciechowski, On the additivity of the Minkowski functionals. Numer. Funct. Anal. Optim. 34 (2013), 635-647.

[22] W. A. Kirk, Fixed points of asymptotic contractions. J. Math. Anal. Appl. 277 (2003), 645-650.

[23] M. A. Krasnosel'skiǔ, G. M. Vaŭnikko, P. P. Zabreı̌ko, Ya. B. Rutitskiǐ and V. Ya. Stetsenko, Approximate Solution of Operator Equations. WoltersNoordhoff Publishing, Groningen, 1972.

[24] G. Kurepa, Tableaux ramifiés d'ensembles. Espaces pseudo-distanciés. C. R. Math. Acad. Sci. Paris 198 (1934), 1563-1565.

[25] A. I. Perov, On the Cauchy problem for a system of ordinary differential equations. Približ. Metod. Rešen. Differencial'. Uravnen. Vyp. 2 (1964), 115-134 (in Russian).

[26] Sh. Rezapour and R. Hamlbarani, Some notes on the paper: "Cone metric spaces and fixed point theorems of contractive mappings" by L.-G. Huang and X. Zhang. J. Math. Anal. Appl. 345 (2008), 719-724.

[27] W. Rudin, Functional Analysis. International Series in Pure and Applied Mathematics, McGraw-Hill, New York, 1991. 
[28] P. P. Zabreǔko, K-metric and K-normed linear spaces: Survey. Collect. Math. 48 (1997), 825-859.

Jacek Jachymski

Institute of Mathematics

Łódź University of Technology

Wólczańska 215

93-005 Łódź

Poland

e-mail: jacek.jachymski@p.lodz.pl

Jakub Klima

Institute of Mathematics

Łódź University of Technology

Wólczańska 215

93-005 Łódź

Poland

e-mail: jakub.klima.87@gmail.com

Open Access This article is distributed under the terms of the Creative Commons Attribution 4.0 International License (http://creativecommons.org/licenses/by/4.0/), which permits unrestricted use, distribution, and reproduction in any medium, provided you give appropriate credit to the original author(s) and the source, provide a link to the Creative Commons license, and indicate if changes were made. 